\title{
ACONDICIONAMIENTO DE LA ARQUERIA DE LOS NUEVOS MINISTERIOS EN EL PASEO DE LA CASTELLANA. MADRID/ESPAÑA
}

\author{
(FITTING UP THE NUEVOS MINISTERIOS ARCADE IN THE PASEO DE LA \\ CASTELLANA)
}

\author{
Ramón Canosa, Javier García Lomas, Eduardo Méndez y Adolfo Morán \\ Arquitectos \\ Gonzalo Cabo de la Sierra \\ Aparejador \\ (149-35)
}

\section{RESUMEN}

Con el fin de recuperar y revalorizar este elemento arquetectónico que limita el gran patio interior de los Nuevos Ministerios en su fachada al Paseo de la Castellana, y que por su especial situación habia quedado prácticamente abandonado, se pensó en utilizarlo como galería de exposiciones.

Para su adaptación a este propósito, se proyectó una solución muy simple basada en el mantenimiento de la arquitectura existente y en la posibilidad de devolver el edificio a su estado primitivo. Con este fin, se han utilizado falsos techos, cerramientos acristalados de carpintería disimulada, e instalaciones ocultas, que son desmontables en su totalidad, y que, en ningún caso, han afectado a la construcción anterior.

\section{SUMMARY}

For the purpose to recover and to revalue this architectural element which limits the inner court of the Nuevos Ministerios with the Paseo de la Castellana, that had remained practically abandoned because of his particular position, it was conceived to use it as an exhibition gallery.

To adapt it for this purpose, a simple solution has been planned, based in the maintenance of the existing architecture, with the possibility to put the building back to its original state. So, false ceilings, glass shutter joinery, hidden equipments has been used, the whole absolutely dismountable and without damage for the original construction.
Por encargo de la Dirección General de Arquitectura y Vivienda se redactó el proyecto de adaptación de la arquería exterior de los Nuevos Ministerios, que cierra la gran plaza en su fachada con el Paseo de la Castellana, para una sala de exposiciones.

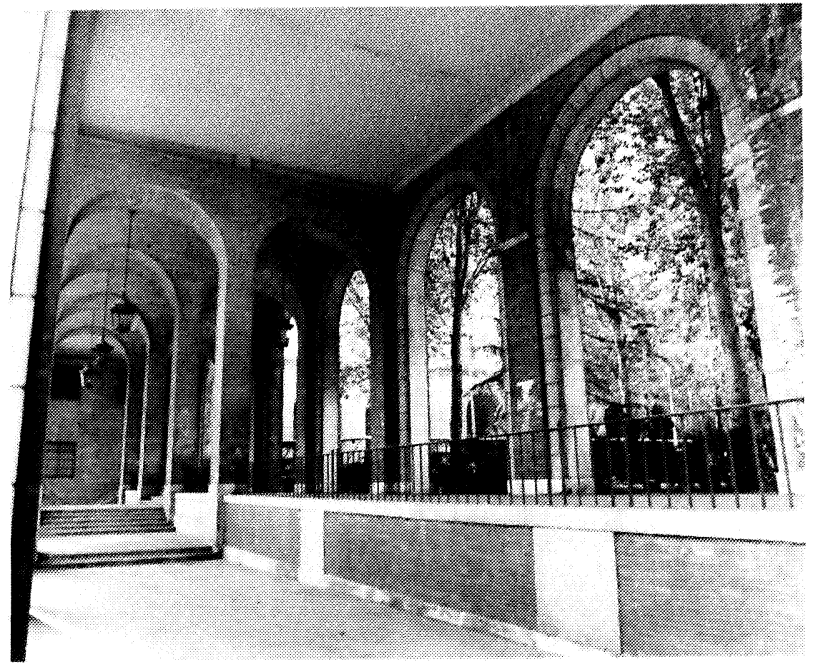

\section{Antecedentes históricos}

El edificio de los Nuevos Ministerios fue proyectado por el arquitecto Secundino Zuazo, para albergar los Ministerios de Gobernación, Obras Públicas y Agricultura.

Las obras se iniciaron durante la Segunda República, siendo paralizadas durante el período de la Guerra Civil española, y reanudadas posteriormente, con arreglo a los primitivos diseños, hasta su terminación en 1958.

La reconstrucción de la arquería que cierra la gran plaza, y que había quedado en estructura y sin construir los arcos interiores en el momento de la paralización de las obras, data, por lo tanto, de esta segunda época.

La arquería está constituida por unos arcos exteriores con una distancia entre ejes de $6,00 \mathrm{~m}$, una luz de $5,00 \mathrm{~m}$, y una altura en la clave, variable según la pendiente, que alcanza hasta los $8,00 \mathrm{~m}$. La luz libre de la arquería es de $9,80 \mathrm{~m}$. 


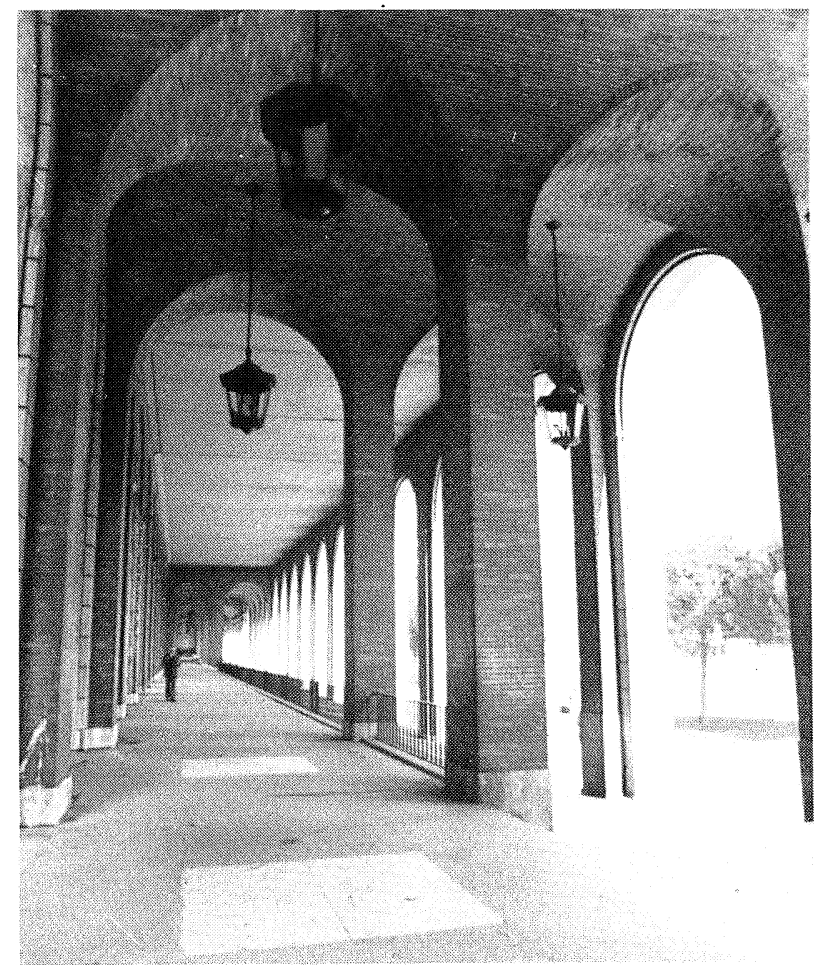

La diferencia de niveles que se produce entre el $\mathrm{Pa}-$ seo de la Castellana, en pendiente, y la zona interior de jardineria, horizontal, se resolvió haciendo coincidir los dos niveles en el centro, para el paso de carruajes, y dividiendo longitudinalmente la galería en dos niveles: uno que se mantiene horizontal siguiendo el plano interior del jardín de la gran plaza, y otro variable que se va adaptando a la pendiente del Paseo de la Castellana.

El conjunto de la arquería está cortado por tres pasos: uno central, de cuatro módulos, para vehículos, y dos laterales, de tres módulos, para peatones. Dejan el paso libre a los jardines desde la Castellana, y sirven de acceso directo a los Ministerios de Obras Públicas y Urbanisno y Trabajo. La estructura de la arquería en estos pasos es distinta, con unos pilares centrales que provocan unas bóvedas de ladrillo visto.

La estructura general de la arquería es de hormigón armado, con piedra de granito y de Colmenar como recubrimiento y en la formación de los arcos. La cubierta está hecha por medio de una losa acartelada, de hormigón armado. Los solados son de granito, con unas barandillas sobre el muro del mismo material, que salvan los desniveles mencionados.

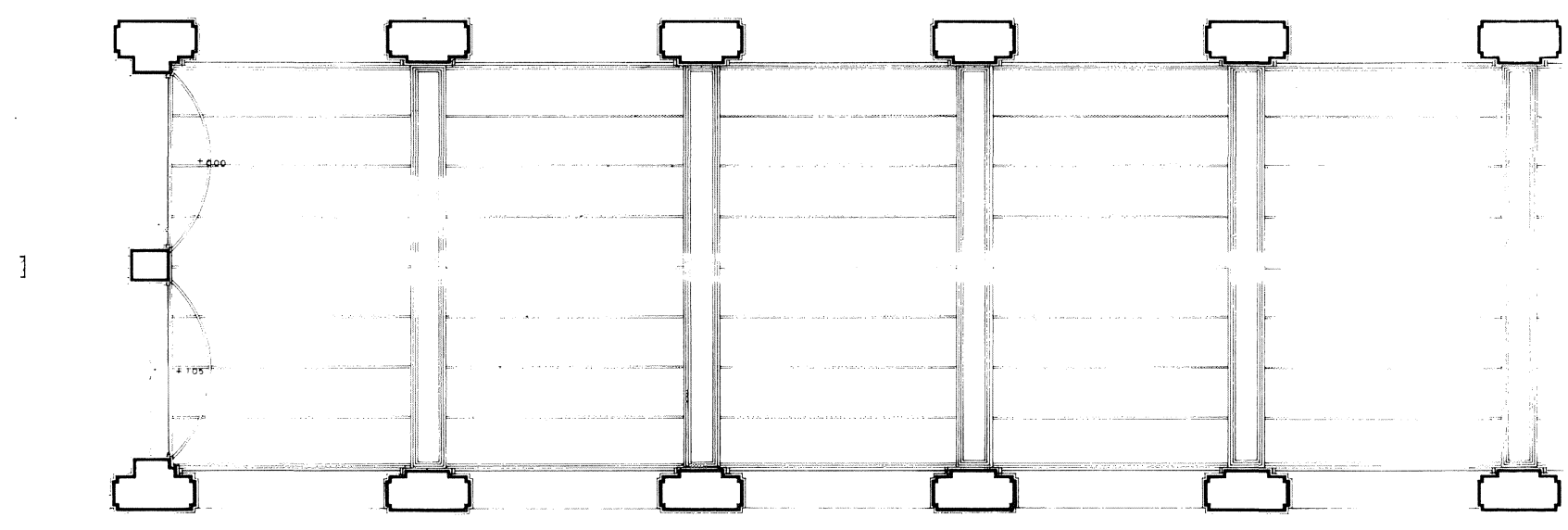

Planta de techos.

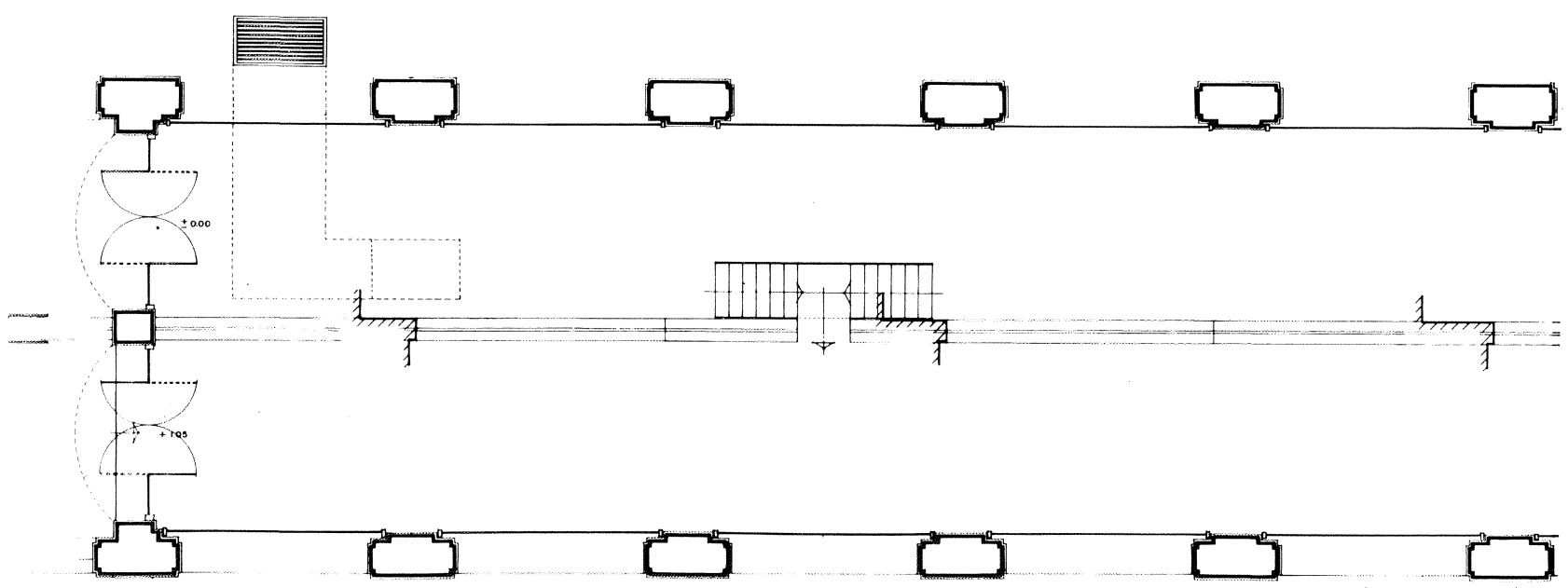




\section{Obras proyectadas}

La solución adoptada se ha desarrollado a partir de la idea fundamental de no variar el carácter de la arquitectura existente, y al mismo tiempo revitalizar una zona que, dadas las especiales características de los juegos de niveles y la jardinería, evitaba el tránsito por la misma, habiéndola dejado en un estado de total abandono.

La solución es muy simple y se limita a cerrar los arcos con lunas, soportadas por una carpintería que queda oculta desde el exterior, y unos techos de plástico en forma de bóveda de cañón seguido, que proporciona una iluminación central ambiental independiente de los focos que se instalen para la iluminación concreta de las obras a exponer.

Para no afectar al juego de luces y sombras de los arcos, se ha retranqueado el plano de cerramiento que, al mismo tiempo, marca el corte entre el material de tratamiento exterior, de piedra, y el del interior, de fábrica de ladrillo que se prolonga formando arcos de un pié de ladrillo a sardinel.

En un principio se pensó en la realización de un acristalamiento sin ningún tipo de perfilería, pero ante la di-

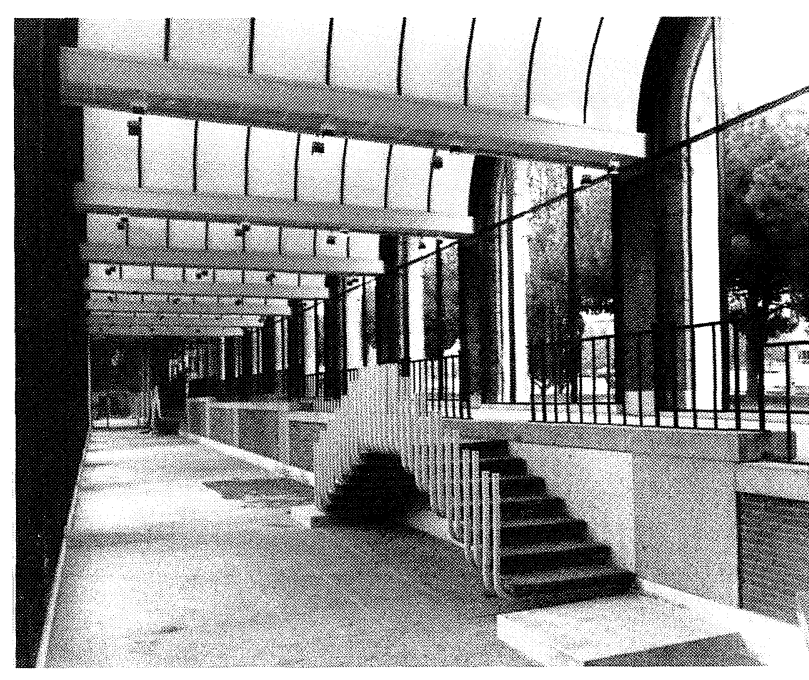

ficultad de realizar esta solución se llegó a reducir la carpintería al mínimo. Esta carpintería, que sigue la forma del arco de medio punto, se adapta al ladrillo, con lo que queda oculta desde el exterior y no destaca en el interior. Abundando aún más en este sentido, se ha empleado una carpintería de aluminio Astrawall, que tiene la característica de que las lunas se reciben

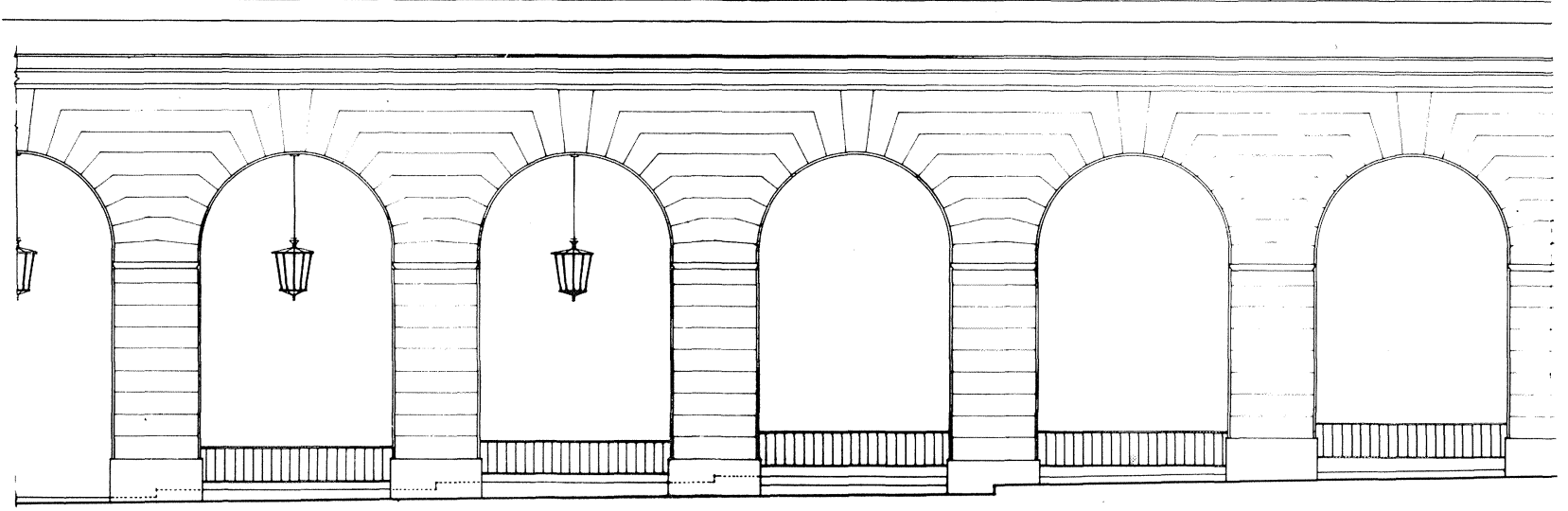

Fachada principal al Paseo de la Castellana.

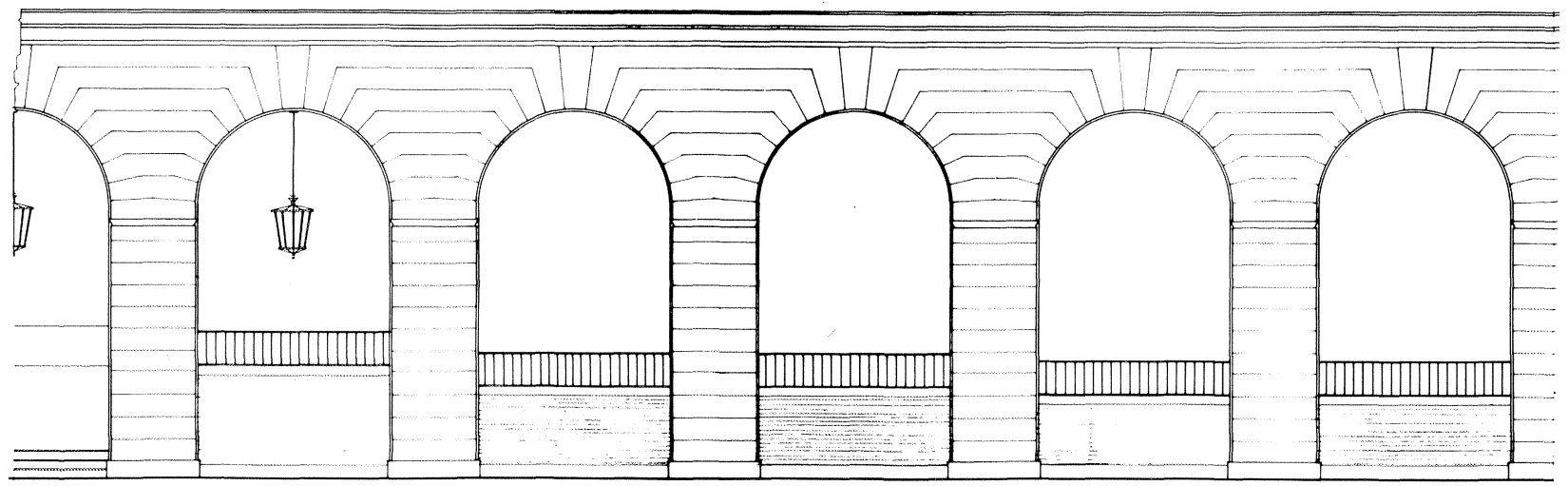

Fachada posterior a jardines. 
con neopreno negro, ocultando totalmente el aluminio. En las zonas en las que parte del aluminio queda visto, se ha anodizado en negro, igualándolo al neopreno.

En el interior no se ha variado el espacio existente con sus distintos niveles, manteniendo la separación longitudinal entre los mismos y conservando el antiguo desnivel para procurar vistas elevadas sobre los objetos a exponer. La comunicación entre las dos zonas se consigue mediante dos escaleras, que han sido simplemente atornilladas como elementos movibles y claramente diferenciados de los elementos de cerrajería existentes.

Para compartimentar este gran espacio longitudinal, el tratamiento de los techos se ha estudiado a base de diez bóvedas de cañón que cortan transversalmente la nave y revalorizan la arquería. Este falso techo ligero, realizado a base de una lámina traslúcida de policarbonato, sirve al mismo tiempo para ocultar las instalaciones de aire acondicionado, luz ambiental, megafonía, instalación eléctrica, etc., siendo visitable para reparaciones o mantenimiento. Queda iluminado por una luz tenue, simplemente ambiental, con focos que reflejan su luz sobre el techo pintado de blanco.

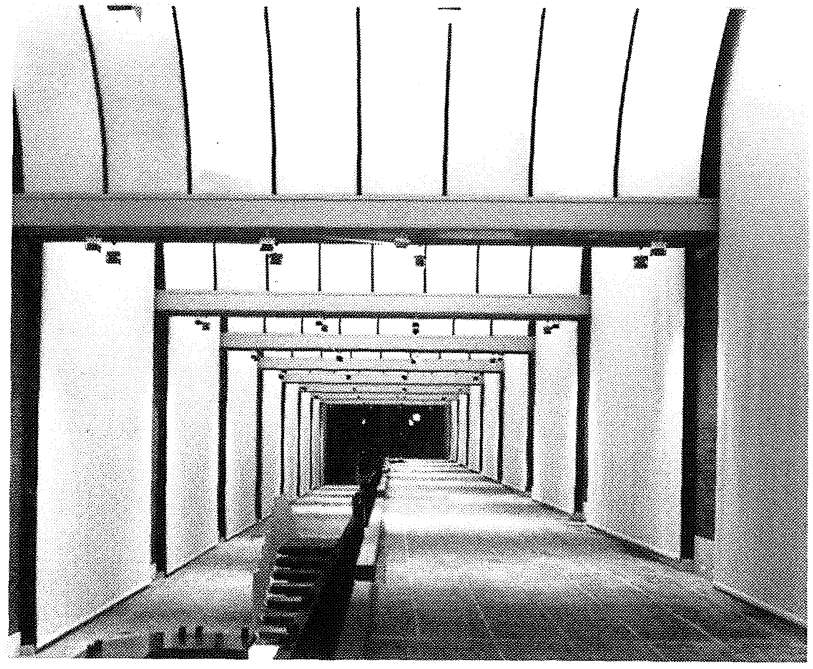

La iluminación propia de la exposición se consigue con focos que corren, entre cada dos bóvedas, por dos carriles electrificados.

El acondicionamiento de aire se obtiene mediante bombas de calor ocultas sobre la cubierta, con taladros en el forjado para el paso de los tubos, que quedan escondidos sobre las bóvedas de plástico.

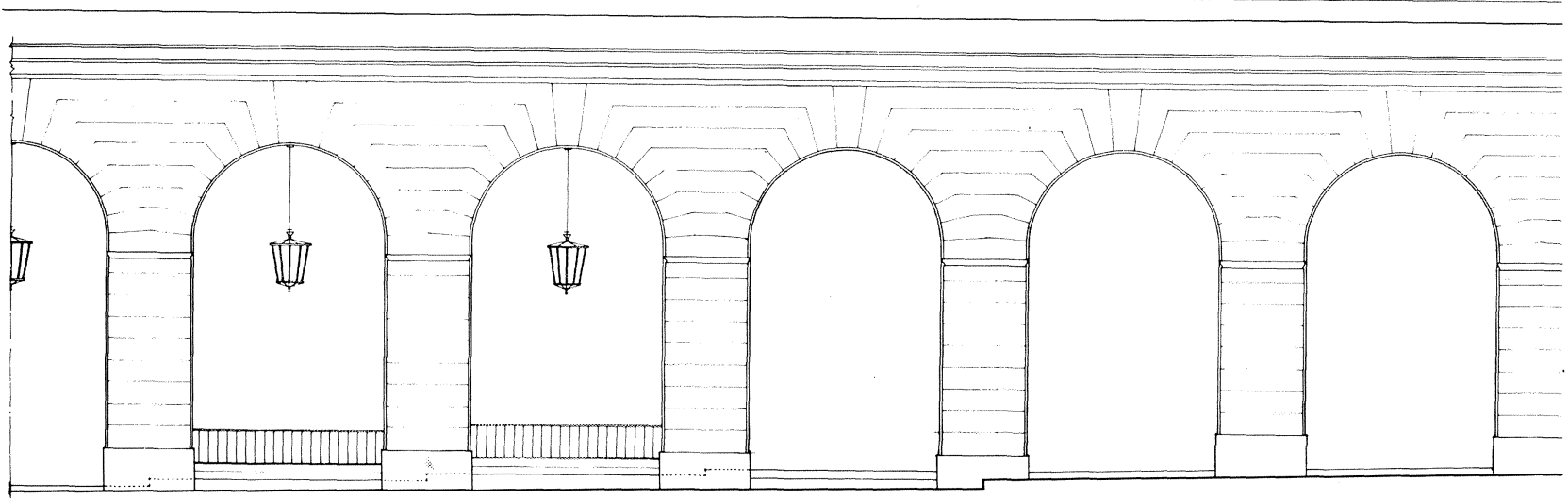

Alzado principal al Paseo de la Castellana.

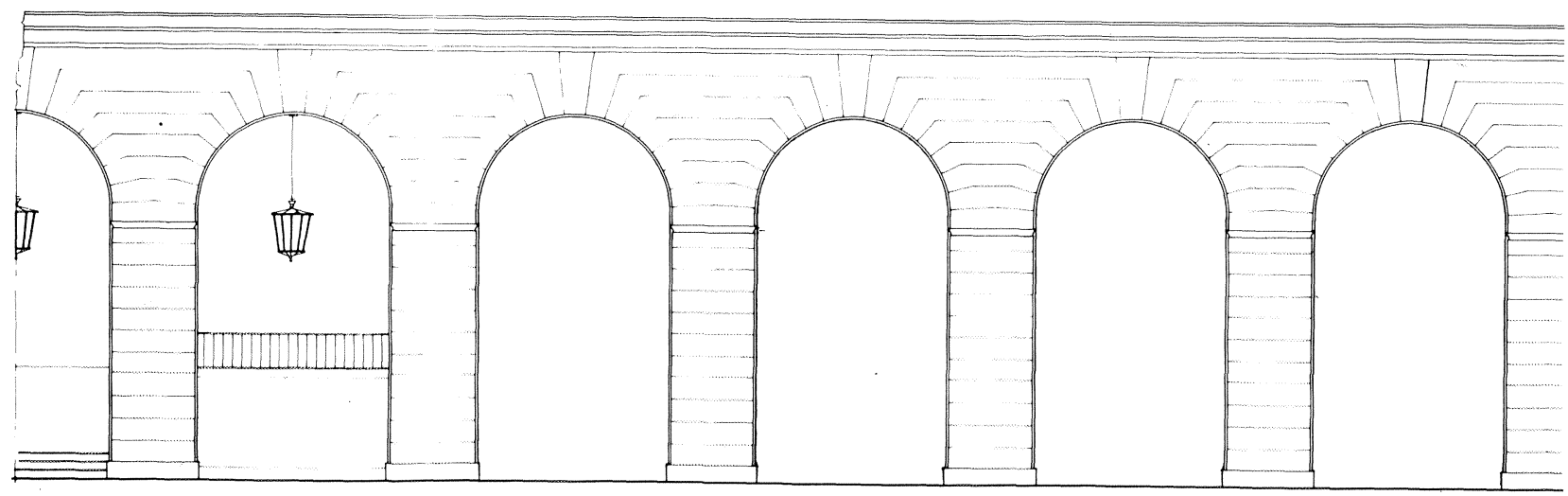

Alzado posterior a jardines. 


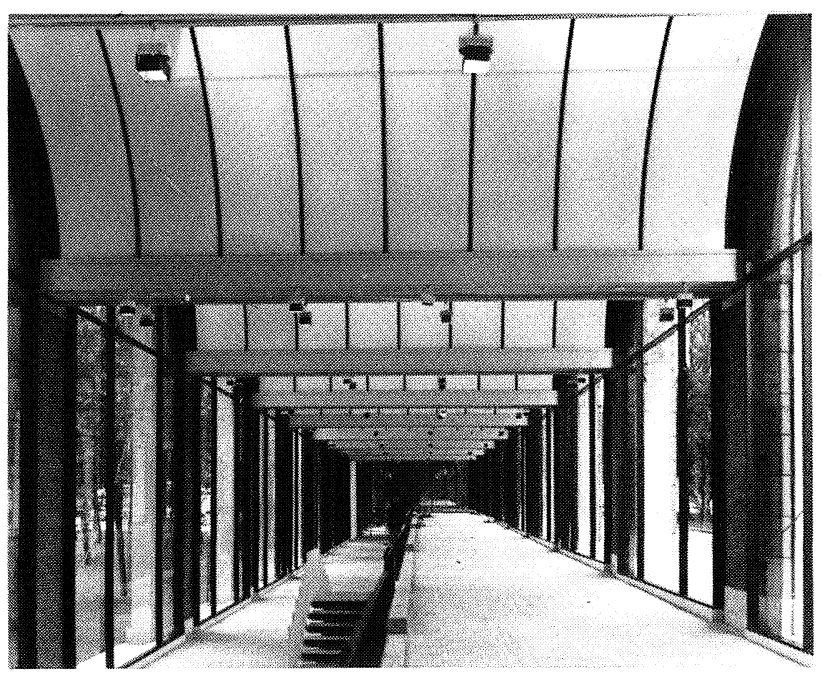

Todos los elementos proyectados, como falso techo, cerramiento, instalaciones, etc., se han concebido de manera que pueden ser desmontados sin que su instalación afecte de modo irreversible o desvirtúe el sentido del edificio, pudiéndose, por tanto, identificar claramente las obras realizadas y al mismo tiempo comprender el estado primitivo. Esto puede verse in situ, ya que la adaptación y aprovechamiento se ha llevado a cabo solamente en una zona de la arquería.

Las instalaciones se han realizado sin efectuar una sola roza en el edificio, estando centralizadas en una consola junto al mostrador de información, desde donde se pueden controlar todos los sistemas instalados.

Todas las conexiones se han realizado desde el falso techo hasta el pupitre de mando, a través de un «árbol eléctrico" exterior al edificio que conecta con un sótano existente bajo la galería, pudiendo, por lo tanto, en todo momento, modificar cualquier tipo de instalación sin ninguna obra de reforma.
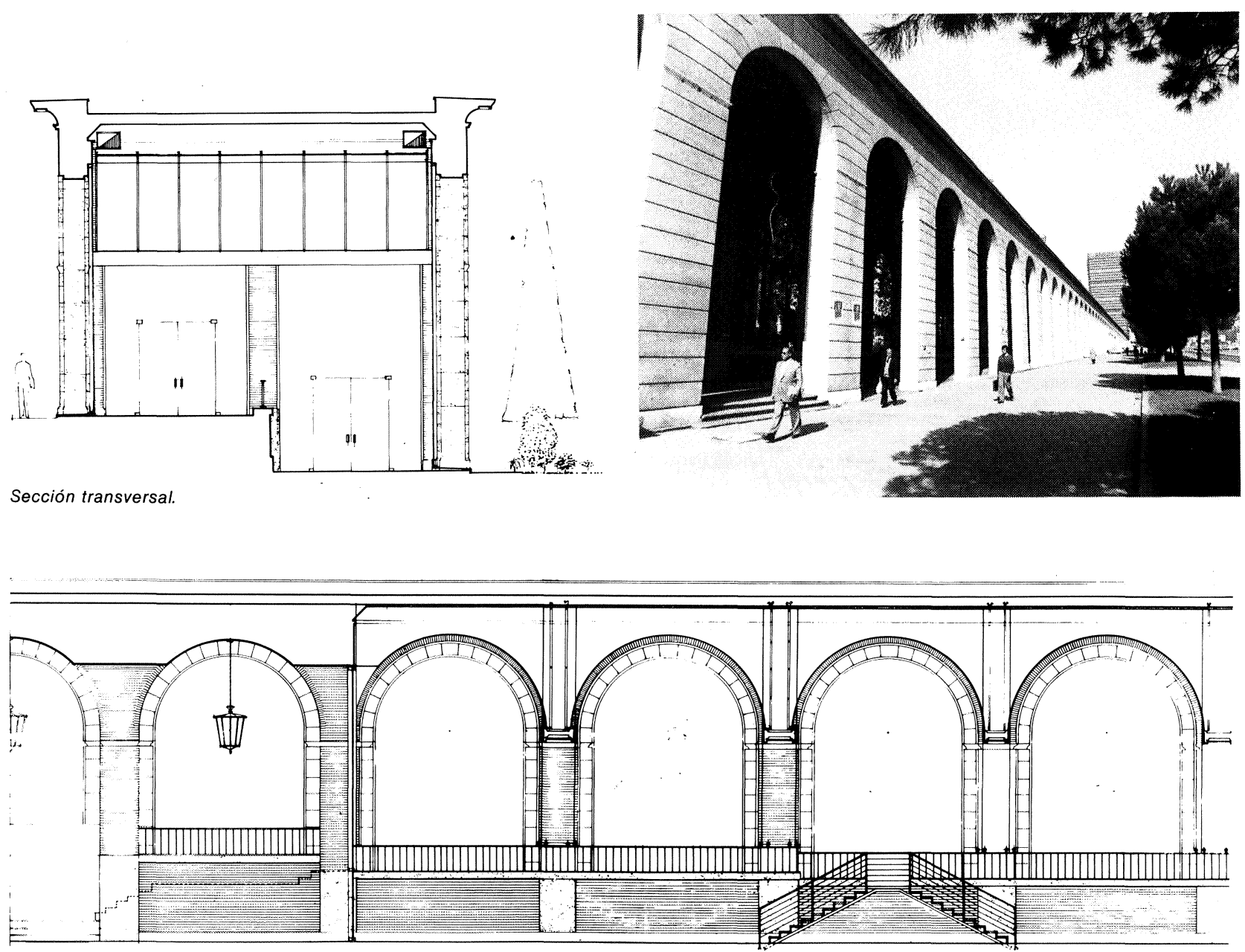

Sección longitudinal. 